\title{
Hysterectomy in Uterus Myomatosus - is the Robot the Jack-of-all-Trades? - A 15-Year Retrospective from a Tertiary Referral Center
}

\author{
Peter Rusch* (D), Rainer Kimmig, Pawel Mach, Sydney S Krolle and Paul Buderath \\ Department of Gynecology and Obstetrics, School of Medicine, University of Duisburg-Essen, Germany
}

\begin{abstract}
Objective: Minimally invasive surgery (MIS) becomes the surgical gold standard for benign hysterectomy. Introduction of robotic-assisted surgery is supposed to increase laparoscopic feasibilities. Debate exists about the relevance of open abdominal hysterectomy and of vaginal routes.
\end{abstract}

Methods: 15-year single center retrospective of all hysterectomies for uterus myomatosus in a tertiary university hospital and high-volume robotic center between 2002 and 2015. Comparison of clinical characteristics, operative outcomes, and complications for four different routes of hysterectomy (abdominal-open,vaginal, laparoscopic, robotic-assisted laparoscopic).

Results: Data from 737 patients were analyzed for peri- and postoperative morbidity according to surgical access for hysterectomy. Minimally-invasive procedures resulted in less postoperative complications and shorter hospital stay than laparotomy. Robotic assistance expanded feasibilities of laparoscopic hysterectomy with regard to comorbidities and size of uterus, thus finally substituting laparotomic and vaginal routes.

Conclusion: This retrospective comparison of four different routes for benign hysterectomy confirmed the known advantages of less morbidity and hospitalization in MIS. Only five years after market launch robotic-assisted laparoscopy obviously extends feasibilities for minimal invasive hysterectomy thus avoiding the risks of open surgery for the vast majority of women. More than ever the indication for open abdominal hysterectomy must be carefully balanced against less invasive surgical alternatives.

\section{Keywords}

Robotic surgery, Laparoscopy, Uterus myomatosus, Fibroid, Hysterectomy

\section{Introduction}

Simple hysterectomy for benign uterine conditions is the second most common surgical procedure in operative gynecology after cesarean section [1]. Indications involve uterine fibroids or adenomyosis uteri, endometriosis or prolapse. Besides, hysterectomy is part of the oncological strategy in malignancies of the uterus and ovaries.

Different surgical approaches exist how to perform hysterectomy: First open abdominal subtotal hysterectomy was performed by Charles Clay in Manchester, England in 1842. For a long time supracervical hysterectomy remained the preferred surgical technique for prevention of peritonitis and reduced risk of urinary tract injury. Due to high mortality rates of $70-90 \%$, abdominal hysterectomy was formally condemned in the interim by the Academy of Medicine in Paris in 1872. Only with the introduction of modern anesthesia, antisepsis and improved surgical instruments Richardson in the United States successfully performed a total abdominal hysterectomy in 1929. Subsequently, total hysterectomy replaced supracervical techniques for the benefit of prevention of cervical cancer. Today, abdominal hysterectomy is still the method of choice in large uteri or in case of complex surgery in the pelvis.

Vaginal hysterectomy dates back to Sopranos of Ephesus $120-Y e a r s$ AD. He removed an inverted uterus that had become gangrenous [2]. The first planned vaginal hysterecto-

* Corresponding author: Peter Rusch, Department of Gynecology and Obstetrics, School of Medicine, University of Duisburg-Essen, Hufelandstr 55, 45122 Essen, Germany

Accepted: July 18, 2020

Published online: July 20, 2020

Citation: Rusch P, Kimmig R, Mach P, et al. (2020) Hysterectomy in Uterus Myomatosus - is the Robot the Jack-of-all-Trades? A 15-Year Retrospective from a Tertiary Referral Center. Adv Laparoscopy 3(1):69-75 
Citation: Rusch P, Kimmig R, Mach P, et al. (2020) Hysterectomy in Uterus Myomatosus - is the Robot the Jack-of-all-Trades? - A 15-Year Retrospective from a Tertiary Referral Center. Adv Laparoscopy 3(1):69-75

my was successfully performed in 1822 by Sauter of Baden, but the patient developed a vesico-vaginal fistula and died 6 months postoperatively [3]. It was only by the 1920s that the operative mortality rates of both vaginal and abdominal hysterectomy were comparable at $2-3 \%$.

Jacobaeus of Stockholm was the first to perform human laparoscopy with pneumoperitoneum in 1911. The German Kurt Semmis deemed the founder of gynecological laparoscopy. He first described a technique for laparoscopic assisted vaginal hysterectomy (LAVH) in 1984. Four years later Harry Reich performed the first total laparoscopic hysterectomy (TLH) in Pennsylvania on a $230 \mathrm{~g}$ fibroid uterus [4]. Since minimal invasive surgery became clinical routine, the technique of conventional straight-stick laparoscopy is still demanding in the context of large uteri and challenging in altered anatomy.

The introduction of robot-assisted surgery is one of the most remarkable technological innovations of the past decades in the field of surgery. Starting with the approval of the daVinci Surgical System (Intuitive Surgical Inc., Sunnyvale, California, USA) by the American Food and Drug Administration (FDA) in 2000, the technique has then been used by an ever-growing number of surgeons in different surgical disciplines throughout the world. The technique is a further development of conventional laparoscopic surgery; while usually positioned beside the patient the surgeon sits comfortably and ergonomically optimized at a console in robotic-assisted laparoscopy. A modern camera with two binoculars provides three-dimensional high-definition vision. Control masters allow ultra-precise motion control of the camera and of up to three wristed instruments by remote control.

The FDA approval for robotic gynecologic surgery followed in 2005. Since then there has been an exponential growth of DaVinci ${ }^{\circledR}$-robotic surgeries with laparoscopic hysterectomy being the largest and fastest growing procedure. 15-Years later evolution of robotic surgery is far from over with multiple serious competitors on the horizon, which will impact the cost-benefit-ratio of the still expensive surgical tool With regard to clinical outcome and while technical improvements and new educational strategies push the limits for minimally invasive surgery, there is debate whether alternative routes - vaginally/open abdominal - still have their place in the context of hysterectomy.

However, most surgeons will agree that in certain situations - i.e. extremely large uteri, multiple previous surgical procedures or comorbidities, especially morbid obesity - A minimally invasive approach is at times challenging or even impossible. As a leading European center for robot-assisted gynecological surgery, we reviewed all our cases of hysterectomy for the indication of uterus myomatosus from 20022015. Our aim was to describe the evolution of robot-assisted surgery in the context of benign hysterectomy in this observational period deriving the answer if there is still a rationale for alternative routes.

\section{Material and Methods}

Digital files and surgical reports of all patients with total hysterectomy for the indication of fibroid uterus between 2002 and 2015 were retrospectively analyzed. The research protocol was approved by the Ethics Committee before the study began. Due to the retrospective character of the study it was not necessary to obtain informed consent of the patients.

The daVinci ${ }^{\circledR}$ robotic system was introduced at our institution in 2010. Data were investigated as to the route of operation (abdominal vs. vaginal vs. laparoscopic vs. robot-assisted laparoscopic hysterectomy), type of hysterectomy (supracervical vs. total), concomitant adnexectomy performed with the hysterectomies, patient characteristics, uterine weight, skin-to-skin time and intra- and postoperative morbidity. Statistical analysis was done using IBM SPSS 24. Statistical significance was defined as $p<0.05$. All operations were performed by gynecologic surgeons well experienced in conventional laparoscopy.

\section{Results}

A total of $n=737$ patients underwent hysterectomy for uterine fibroids during the observational period. Of these, $\mathrm{n}=197$ received open abdominal, $\mathrm{n}=116$ vaginal, $\mathrm{n}=173$ laparoscopic and $n=251$ robotic-assisted laparoscopic hysterectomy. BMI and age did not differ significantly between groups. Table 1 shows the demographic characteristics of the collective.

Skin-to-skin time, hemoglobin (hb)-decrease and hospital stay differed significantly between the surgical approaches. Skin-to-skin time was longest in robotic surgery, while patients with abdominal hysterectomy had the longest hospital stay and greatest decrease in hb-levels.

Uni- or bilateral salpingo-ovarectomy was performed in 153 cases (20.7\%). Rates were significantly different between the surgical approaches. While in abdominal approaches $64.2 \%$ patients received removal of adnexa, this was the case only in $2.6 \%$ of vaginal hysterectomies. Rates did not

Table 1: Demographic data; Data presented as mean $+/-$ SD, median (minimum: maximum) or $n$ (\%). Statistical comparison of medians was performed by Kruskal-Wallis-test for independent samples.

\begin{tabular}{|l|l|l|l|l|l|}
\hline $\begin{array}{l}\text { Demographic } \\
\text { data }\end{array}$ & $\begin{array}{l}\text { Abdominal } \\
\text { hysterectomy (AH) }\end{array}$ & $\begin{array}{l}\text { Vaginal Hysterectomy } \\
\text { (VH) }\end{array}$ & $\begin{array}{l}\text { Laparoscopic } \\
\text { Hysterectomy (LH) }\end{array}$ & $\begin{array}{l}\text { Robot-assisted } \\
\text { laparoscopic } \\
\text { Hysterectomy (RALH) }\end{array}$ \\
\hline Age & $46.7(31-85 ; 6.6)$ & $47.2(36-75 ; 7.7)$ & $46.9(33-73 ; 7.0)$ & $47.8(31-75 ; 6.5)$ & 0.165 \\
\hline BMI & $26.7(18.2-51.7 ; 6.4)$ & $27.4(19.4-44.3 ; 5.6)$ & $25.4(19.0-48.0 ; 5.1)$ & $27.9(17.3-66.4 ; 7.5)$ & 0.062 \\
\hline Gravida & $1.32(0-7 ; 1.5)$ & $2.7(0-8 ; 1.8)$ & $1.8(0-5 ; 1.4)$ & $1.84(0-10 ; 1.8)$ & $<0.001$ \\
\hline Parity & $1.0(0-4 ; 1.1)$ & $1.92(0-6 ; 1.2)$ & $1.42(0-6 ; 1.2)$ & $1.4(0-6 ; 1.23)$ & $<0.001$ \\
\hline
\end{tabular}


Citation: Rusch P, Kimmig R, Mach P, et al. (2020) Hysterectomy in Uterus Myomatosus - is the Robot the Jack-of-all-Trades? - A 15-Year Retrospective from a Tertiary Referral Center. Adv Laparoscopy 3(1):69-75

Table 2: Operative data of the patients according to surgical route for hysterectomy. Data are presented as mean (minimum-maximum; SD). Statistical comparison of medians was performed by Kruskal-Wallis-test for independent samples. Comparison of frequencies (salpingooopherectomy) was performed by Chi-square-test.

\begin{tabular}{|c|c|c|c|c|c|}
\hline Operative Data & $\begin{array}{l}\text { Abdominal } \\
\text { hysterectomy }(\mathrm{AH})\end{array}$ & $\begin{array}{l}\text { Vaginal } \\
\text { Hysterectomy (VH) }\end{array}$ & $\begin{array}{l}\text { Laparoscopic } \\
\text { Hysterectomy (LH) }\end{array}$ & $\begin{array}{l}\text { Robot-assisted } \\
\text { laparoscopic } \\
\text { Hysterectomy (RALH) }\end{array}$ & P-value \\
\hline Skin-to-skin time (min) & $109.2(29-237 ; 42.6)$ & $87.4(15-215 ; 35.8)$ & $121.8(49-610 ; 57.2$ & $130.6(50-470 ; 56.3)$ & $<0.001$ \\
\hline $\mathrm{Hb}$ decrease $(\mathrm{g} / \mathrm{dl})$ & $2.08(-1-6.6 ; 1.4)$ & $1.72(-2.8-6.6 ; 1.2)$ & $1.75(-4.5-6.0 ; 1.3)$ & $1.6(-1.6-8.7 ; 1.1)$ & $<0.001$ \\
\hline Hospital Stay (d) & $8.7(4-25 ; 3.6)$ & $6.5(2-15 ; 2.3)$ & $5.58(1-14 ; 2.1)$ & $5.22(2-32 ; 2.4)$ & $<0.001$ \\
\hline Specimen weight (g) & 585 (17-9500; 919) & $183(25-741 ; 112)$ & $225(38-729 ; 137)$ & 355 (55-2300; 294) & $<0.001$ \\
\hline Salpingo-oopherectomy & $61(31.0 \%)$ & $3(2.6 \%)$ & $41(24.1 \%)$ & $48(19.1 \%)$ & $<0.001$ \\
\hline
\end{tabular}

Table 3: Complications.

\begin{tabular}{|c|c|c|c|c|c|}
\hline & $\begin{array}{l}\text { Abdominal } \\
\text { hysterectomy } \\
\text { (AH) }\end{array}$ & $\begin{array}{l}\text { Vaginal } \\
\text { Hysterectomy } \\
\text { (VH) }\end{array}$ & $\begin{array}{l}\text { Laparoscopic } \\
\text { Hysterectomy } \\
\text { (LH) }\end{array}$ & $\begin{array}{l}\text { Robot-assisted } \\
\text { laparoscopic } \\
\text { Hysterectomy (RALH) }\end{array}$ & P-value \\
\hline Intraoperative complications (\%) & $2(1.0)$ & $1(0.9)$ & $3(1.7)$ & $2(0.8)$ & 0.81 \\
\hline Postoperative complications (\%) & $36(18.3)$ & $10(8.6)$ & $13(7.5)$ & $21(8.4)$ & 0.002 \\
\hline$\geq$ Grade $3(\%)$ & $10(5.1)$ & $2(1.7)$ & $4(2.3)$ & $5(2.0)$ & 0.18 \\
\hline Conversion to laparotomy & - & 0 & $3(1.7 \%)$ & $2(0.8 \%)$ & 0.17 \\
\hline
\end{tabular}

differ significantly between open abdominal hysterectomy (31\%), conventional laparoscopy $(24.1 \%)$ and robotic surgery (19.1\%).

Size of uterus did not differ significantly between groups. Operative data are shown in Table 2.

Intra-operative complications were rare and not significantly different between the surgical approaches. Altogether, eight intra-operative complications occurred in the whole cohort of 737 hysterectomies, seven of these were iatrogenic bladder lesions. One other intra-operative complication was a circum script laceration of the vaginal skin caused by retrieval of the specimen in a robotic case.

However, open abdominal surgery had a significantly higher rate of postoperative complications (18.3\%) than the other three routes with rates around $8 \%$. Severe complications (Clavien-Dindo $\geq 3$ ) [5] were more than twice as frequent in abdominal hysterectomy compared to the three other approaches, but this did not reach statistical significance. Conversion to laparotomy was extremely rare in robotic surgery and in conventional laparoscopy with $0.8 \%$ and $1.7 \%$ of cases, respectively. An overview of the complication rates is given in Table 3.

To assess the influence of BMI on intra- and postoperative complications, we defined two BMI-groups of $<25 \mathrm{~kg} / \mathrm{m}^{2}$ and $>25 \mathrm{~kg} / \mathrm{m}^{2}$ according to the definition of normal body weight of the World Health Organization (WHO) [6]. Analysis via chisquare-test did not reveal any significant differences in either group nor in the whole collective.

Correlation of skin-to-skin time, of hb-decrease and of length of hospital stay with the two BMI groups showed a significant difference only for skin-to-skin time (111 vs. $125 \mathrm{~min}$; t-test $p=0.013$ ) irrespective of route of hysterectomy. This effect of BMI on surgical time remained significant for every subgroup when analyzing the surgical approach separately. No significant relation between BMI neither with blood loss nor with hospital stay was observed. Patient age at surgery did not significantly influence any perioperative variable.

Over the course of the selected time period, we observed a significant shift in the surgical methods with a trend towards minimally-invasive surgery. While the rate of open abdominal hysterectomies decreased from $56.8 \% 2002$ to $1.5 \%$ in 2014 and finally $0 \%$ in 2015, the use of minimally-invasive techniques increased continually over time. In $2013,86.5 \%$ of patients received robotic hysterectomy. While only $43.2 \%$ of patients had minimally-invasive hysterectomy in 2012 none had open abdominal hysterectomy in 2015.

Table 4 shows the absolute numbers and percentages of the different surgical methods for each year. (Figure 1) visualizes the development of surgical techniques over the years 2002-2015.

\section{Discussion}

We herein present the perioperative data of 737 patients with hysterectomy for uterus myomatosus at the Department of Gynecology and Obstetrics at Medical School of University Duisburg-Essen between 2002-2015. Demographic characteristics did not differ significantly between operative subgroups, thus analysis of intra- and postoperative morbidity was applicable for the different routes of hysterectomy. The surgical outcome data and conversion rates of our collective was in line with results of other publications $[7,8]$.

Regarding the route of hysterectomy are levant shift towards minimal invasive surgery favoring robotic surgery was described by several authors $[9,10]$. Accordingly, we detected a massive increase in robotic and thus minimally-invasive 
Citation: Rusch P, Kimmig R, Mach P, et al. (2020) Hysterectomy in Uterus Myomatosus - is the Robot the Jack-of-all-Trades? - A 15-Year Retrospective from a Tertiary Referral Center. Adv Laparoscopy 3(1):69-75

Table 4: Absolute and relative numbers of different surgical routes for hysterectomy.

\begin{tabular}{|c|c|c|c|c|c|c|c|}
\hline \multicolumn{3}{|c|}{ route of hysterectomy } & \multirow{2}{*}{$\begin{array}{l}\text { Open } \\
\text { abdominal } \\
21 \\
\end{array}$} & \multirow{2}{*}{\begin{tabular}{|l|} 
Vaginal \\
5
\end{tabular}} & \multirow{2}{*}{$\begin{array}{l}\text { Conventional } \\
\text { laparoscopy } \\
11\end{array}$} & \multirow{2}{*}{\begin{tabular}{|l|}
$\begin{array}{l}\text { Robot-assisted } \\
\text { laparoscopy }\end{array}$ \\
0
\end{tabular}} & \multirow{2}{*}{\begin{tabular}{|l|} 
total \\
37
\end{tabular}} \\
\hline \multirow[t]{28}{*}{ Year } & \multirow[t]{2}{*}{2002} & $\mathrm{~N}$ & & & & & \\
\hline & & $\%$ & $56.80 \%$ & $13.50 \%$ & $29.70 \%$ & $0 \%$ & $100 \%$ \\
\hline & \multirow[t]{2}{*}{2003} & $\mathrm{~N}$ & 14 & 13 & 6 & 0 & 33 \\
\hline & & $\%$ & $42.40 \%$ & $39.40 \%$ & $18.20 \%$ & $0 \%$ & $100 \%$ \\
\hline & \multirow[t]{2}{*}{2004} & $\mathrm{~N}$ & 18 & 15 & 5 & 0 & 38 \\
\hline & & $\%$ & $47.40 \%$ & $39.50 \%$ & $13.20 \%$ & $0 \%$ & $100 \%$ \\
\hline & \multirow[t]{2}{*}{2005} & $\mathrm{~N}$ & 18 & 11 & 12 & 0 & 41 \\
\hline & & $\%$ & $43.90 \%$ & $26.80 \%$ & $29.30 \%$ & $0 \%$ & $100 \%$ \\
\hline & \multirow[t]{2}{*}{2006} & $\mathrm{~N}$ & 12 & 12 & 12 & 0 & 36 \\
\hline & & $\%$ & $33.30 \%$ & $33.30 \%$ & $33.30 \%$ & $0 \%$ & $100 \%$ \\
\hline & \multirow[t]{2}{*}{2007} & $\mathrm{~N}$ & 19 & 9 & 19 & 0 & 47 \\
\hline & & $\%$ & $40.40 \%$ & $19.10 \%$ & $40.40 \%$ & $0 \%$ & $100 \%$ \\
\hline & \multirow[t]{2}{*}{2008} & $\mathrm{~N}$ & 15 & 15 & 12 & 0 & 42 \\
\hline & & $\%$ & $35.70 \%$ & $35.70 \%$ & $28.60 \%$ & $0 \%$ & $100 \%$ \\
\hline & \multirow[t]{2}{*}{2009} & $\mathrm{~N}$ & 25 & 12 & 30 & 0 & 67 \\
\hline & & $\%$ & $37.30 \%$ & $17.90 \%$ & $44.80 \%$ & $0 \%$ & $100 \%$ \\
\hline & \multirow[t]{2}{*}{2010} & $\mathrm{~N}$ & 22 & 9 & 30 & 12 & 73 \\
\hline & & $\%$ & $30.10 \%$ & $12.30 \%$ & $41.10 \%$ & $16.40 \%$ & $100 \%$ \\
\hline & \multirow[t]{2}{*}{2011} & $\mathrm{~N}$ & 13 & 7 & 19 & 62 & 101 \\
\hline & & $\%$ & $12.90 \%$ & $6.90 \%$ & $18.80 \%$ & $61.40 \%$ & $100 \%$ \\
\hline & \multirow[t]{2}{*}{2012} & $\mathrm{~N}$ & 12 & 5 & 6 & 40 & 63 \\
\hline & & $\%$ & $19.00 \%$ & $7.90 \%$ & $9.50 \%$ & $63.50 \%$ & $100 \%$ \\
\hline & \multirow[t]{2}{*}{2013} & $\mathrm{~N}$ & 7 & 0 & 3 & 64 & 74 \\
\hline & & $\%$ & $9.50 \%$ & $0 \%$ & $4.10 \%$ & $86.50 \%$ & $100 \%$ \\
\hline & \multirow[t]{2}{*}{2014} & $\mathrm{~N}$ & 1 & 3 & 4 & 57 & 65 \\
\hline & & $\%$ & $1.50 \%$ & $4.60 \%$ & $6.20 \%$ & $87.70 \%$ & $100 \%$ \\
\hline & \multirow[t]{2}{*}{2015} & $N$ & 0 & 0 & 4 & 16 & 20 \\
\hline & & $\%$ & $0 \%$ & $0 \%$ & $20.00 \%$ & $80.00 \%$ & $100 \%$ \\
\hline \multirow[t]{2}{*}{ Total } & & $\mathrm{N}$ & 197 & 116 & 173 & 251 & 737 \\
\hline & & $\%$ & $26.70 \%$ & $15.7 \%$ & $23.50 \%$ & $34.10 \%$ & $100 \%$ \\
\hline
\end{tabular}

procedures over time. It was only 5-years after robotic surgery entered our clinic in $\mathbf{2 0 1 0}$ when robotic-assisted minimal invasive hysterectomy completely replaced open abdominal and vaginal approaches.

Coincidentally it was at that time when evidence indicated a benefit of opportunistic salpingectomy during hysterectomy to reduce the hereditary risk of high-grade serous ovarian or peritoneal cancers. While guidelines do not recommend to alter the intended route of hysterectomy for opportunistic salpingectomy [11] patients should be counselled about the possibility that this may not be feasible due to prior adnexal or abdominal surgery; this fact may have biased trends discriminating against vaginal hysterectomy.

Operative time was longest in robotically-assisted surgery. While this might be due to the longer setup-time for the robotic system it can also be the result of the second highest mean specimen weight in the robotic subgroup with only higher weights in the open route-subgroup.

In line with other publications [12] we found significantly shorter hospitalizations for minimal invasive and vaginal hysterectomy compared to open abdominal surgery with the shortest length of stay in the robotic subgroup.

Accordingly, postoperative complications were also rare in minimally-invasive surgery and vaginal hysterectomy compared to open abdominal surgery $[13,14]$.

More than others obese patients seem to be qualified for minimally-invasive procedures because of increased perioperative risks of laparotomy and prolonged hospital stay. At the same time, vaginal hysterectomy or conventional laparoscopy can be challenging in these patients due to physical 


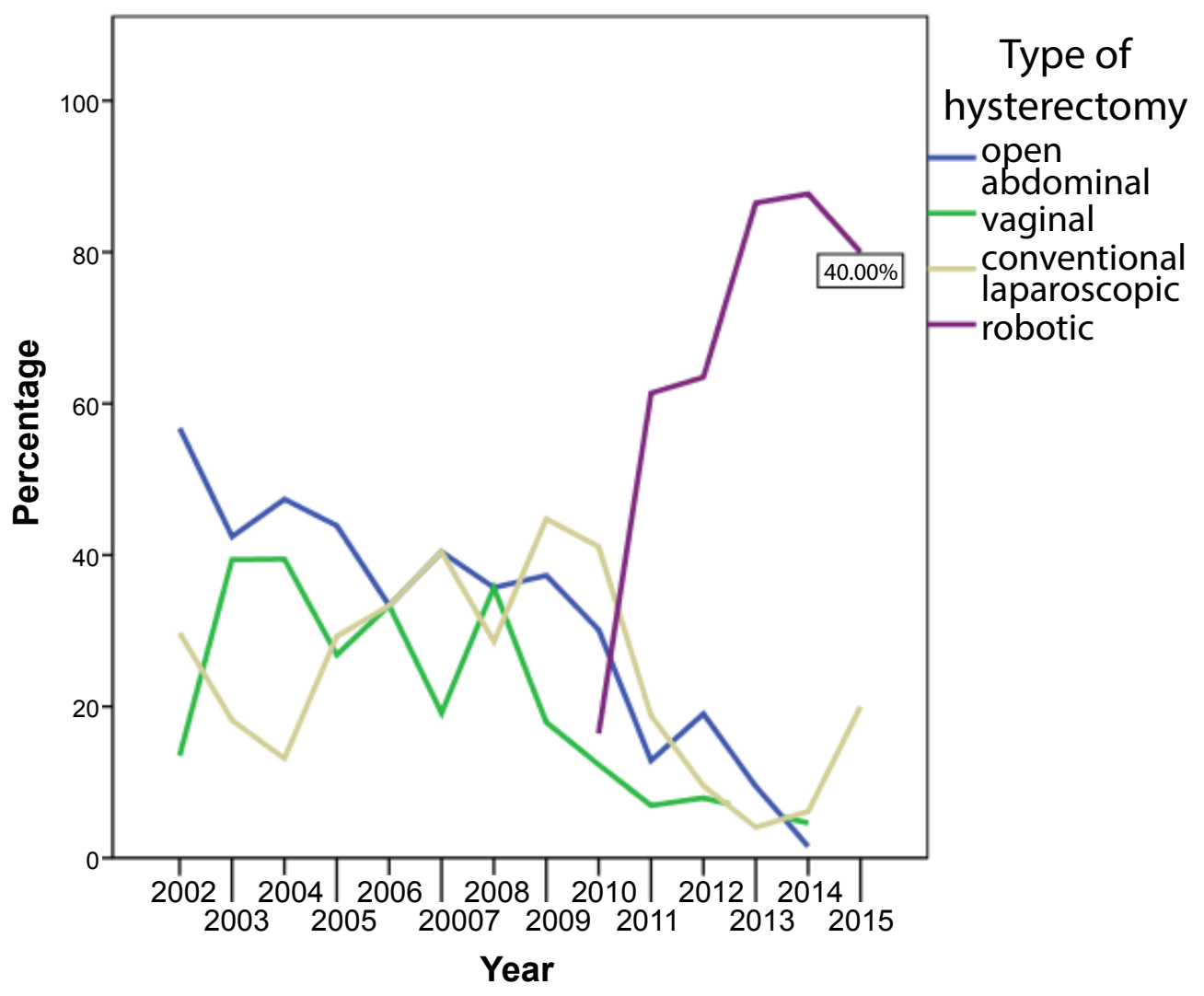

Figure 1: Development of the frequency of surgical techniques over the years 2002-2015.

limitations of redundant vaginal sidewall tissue and thick abdominal wall. The robotic platform may facilitate such surgeries with improved, ergonomic surgeon posture during surgery [15] while at the same time reducing trauma on the abdominal wall and dislocation of trocars, as robotic arms pivoted at the incision sites do not move, but only rotate around a fixed remote center [16].

In our collective the highest BMI of $66.4 \mathrm{~kg} / \mathrm{m}^{2}$ was found in the robotic subgroup, while the second highest BMI $(48.0$ $\mathrm{kg} / \mathrm{m}^{2}$ ) was found in conventional laparoscopy. Subgroups were not different with regard to patients' age at surgery. This together may be indicative of the increased feasibilities of robotic assistance in minimally invasive hysterectomy even in patients being at risk due to severe obesity $[17,18]$ or age-related co-morbidities $[19,20]$. This is of interest especially in the context of substantial obesity rates and a rising life-expectancy in developed countries.

While it seems obvious that natural limits exist for minimally invasive hysterectomy, the question arises if there are definite limits for robot-assisted minimal invasive hysterectomy according to size and weight of the uterus [21]. While abdominal surgery may still be considered by some as the method of choice in extremely large uteri, minimally-invasive hysterectomy is close on its heels. A US study reports the case of a total laparoscopic hysterectomy on a giant uterus weighting more than $3000 \mathrm{~g}$ [22].

In this work, decision about route of hysterectomy was not influenced by BMI or size of uterus. Size of specimen did not differ significantly between groups. In open abdominal hysterectomy mean uterus weight was $585 \mathrm{~g}$, with the largest uterus being $9.5 \mathrm{~kg}$. Interestingly robotic hysterectomy was feasible up to a specimen weight of $2.3 \mathrm{~kg}$, which was three times the maximum weight in conventional laparoscopy $(729$ g). The two largest uteri removed by open abdominal surgery were $4.4 \mathrm{~kg}$ in 2003 and $9.5 \mathrm{~kg}$ in 2004, respectively. At that time robotic surgery was not even available; it is unclear whether or not robotic hysterectomy would have been feasible in these situations, too. At least there is no doubt that robotic surgery took over a relevant proportion of hysterectomies formally performed by one of the alternative routes (i.e. conventional laparoscopy or open abdominal hysterectomy).

Obviously, patient age, BMI, size and weight of the uterus are no valid benchmarks to decide the route of hysterectomy. Considering the technical features and intuitive operability of robotic systems with its effects on learn ability, robotic assistance may even push the limits for minimally-invasive hysterectomy. Thus, it remains in the responsibility of the dedicated surgeon to decide when robot-assisted laparoscopic hysterectomy is preferable, while it is feasible and most beneficial for the individual patient at the same time.

Discussion focuses on validation of proficiency and credentialing for the safe use of the robot on the patient. At the time of our observational period no educational curricula existed, thus it was appropriate to involve surgeons for robotic surgerty who were intensively trained in the field of conventional laparoscopy and open abdominal surgery. 
Citation: Rusch P, Kimmig R, Mach P, et al. (2020) Hysterectomy in Uterus Myomatosus - is the Robot the Jack-of-all-Trades? - A 15-Year Retrospective from a Tertiary Referral Center. Adv Laparoscopy 3(1):69-75

According to literature we felt that the learning curve of robotic surgery was steeper than that of laparoscopy, especially with regard to complex surgical tasks like suturing [23]. Additionally, patients chosen for robotic surgery were more complex with regard to higher BMI and specimen weight than those managed by conventional laparoscopy. The fact that we performed twice as many robotic-assisted hysterectomies since the introduction of the daVinci as by conventional laparoscopy in 14 years may be one expression of the intuitive learnability of the robotic procedure.

Today there is no doubt about the need for urgent implementation of validated educational curricula. This is important from a medico-legal point of view and it is fundamental for the acquisition of valuable and comparable scientific surgical data. The robotic setting facilitates surgical education with the integration of virtual reality training and/or supervision of the procedure by an experienced proctor in a dual-console-setting, thus being implemented in educational curricula [24].

Use of a state-of-the-art laparoscopic equipment - i.e. robotics -increases feasibilities of MIS for hysterectomy. Robotic assistance pushes the limits of conventional laparoscopy and helps to abandon open abdominal hysterectomy for the vast majority of patients. This holds true even in difficult cases with extremely high BMI or large uteri and is irrespective of patient age and age-related co-morbidities [16,25].

With regard to morbidity and mortality any decision about open hysterectomy should be thoroughly balanced against minimal invasive approaches. If in doubt about feasibility in an individual case, it seems reasonable to refer patients to experienced and well-equipped robotic centers.

Guidelines for credentialing robotic centers and for privileging surgeons already exist [24], but still they have to be urgently implemented as quality benchmarks.

Minimally invasive hysterectomy turns out to be the gold standard with regard to clinical outcome. Full evaluation of pelvic anatomy and combination with adnexal surgery favors MIS instead of vaginal hysterectomy. Yet still situations exist, when there is no alternative to laparotomy, thus surgical knowledge of open hysterectomy remains crucial. In this regard open abdominal surgery remains a mainstay and it is also the backbone for safe MIS, if conversion is inevitable for emergency reasons.

\section{Conflict of Interest}

The authors declare that they have the following conflicts of interest:

PR: Reports personal fees from Medtronic, honoraria from Roche and Astra Zeneca, outside the submitted work.

RK: Reports personal fees from Astra Zeneca, personal fees from Roche, personal fees from Pro Strakan (now: Kyowa Kirin), personal fees from Riemser, personal fees from Teva, grants from Intuitive Surgery Inc., outside the submitted work.

PM: Has no conflict of interest.
SSK: Has no conflict of interest.

PB: Reports honoraria from Roche and Astra Zeneca, travel and Congress support from Roche and Pharma Mar, outside the submitted work.

\section{Author Contributions}

PR: conceptualization, investigation, methodology, data collection, analysis, project administration, writing/editing (original/final draft); RK: Supervision, review; PM: Review; SSK: Data collection; PB: Conceptualization, investigation, methodology, data collection, analysis, writing/editing (original/final draft).

\section{References}

1. Neis KJ, Zubke W, Fehr M, et al. (2016) Hysterectomy for benign uterine disease. Dtsch Arztebl Int 113: 242-249.

2. Sutton C (1997) Hysterectomy: A historical perspective. Baillières Clin Obstet Gynaecol 11: 1-22.

3. Bulimar V, Costea I, Glod M, et al. (2016) Implications of hysterectomy on vaginal route in gynecologic pathology. Rev Med Chir Soc Med Nat lasi 120: 581-586.

4. Sparic R, Hudelist G, Berisavac M, et al. (2011) Hysterectomy throughout history. Acta Chir lugosl 58: 9-14.

5. Dindo D, Demartines N, Clavien PA (2004) Classification of surgical complications: A new proposal with evaluation in a cohort of 6336 patients and results of a survey. Ann Surg 240: 205-213.

6. Jan A, Weir C (2019) BMI classification percentile And Cut Off Points.

7. David-Montefiore E, Rouzier R, Chapron C, et al. (2007) Surgical routes and complications of hysterectomy for benign disorders: A prospective observational study in French university hospitals. Hum Reprod 22: 260-265.

8. Chen $\mathrm{CH}$, Chen HH, Liu WM (2017) Complication reports for robotic surgery using three arms by a single surgeon at a single institution. J Minim Access Surg 13: 22-28.

9. Cure N de, Robson SJ (2018) Changes in hysterectomy route and adnexal removal for benign disease in Australia 2001-2015: A national population-based study. Minim Invasive Surg 2018: 5828071.

10. Lenihan JP, Kovanda C, Seshadri Kreaden U (2008) What is the learning curve for robotic assisted gynecologic surgery? J Minim Invasive Gynecol 15: 589-594.

11. (2019) ACOG Committee Opinion No. 774: Opportunistic salpingectomy as a strategy for epithelial ovarian cancer prevention. Obstet Gynecol 133: 279-284.

12. Dubeshter B, Angel C, Toy E, et al. (2013) Current role of robotic hysterectomy. J Gynecol Surg 29: 174-178.

13. Beck TL, Schiff MA, Goff BA, et al. (2018) Robotic, laparoscopic, or open hysterectomy: Surgical outcomes by approach in endometrial cancer. J Minim Invasive Gynecol 25: 986-993.

14. Lim PC, Crane JT, English EJ, et al. (2016) Multicenter analysis comparing robotic, open, laparoscopic, and vaginal hysterectomies performed by high-volume surgeons for benign indications. Int J Gynaecol Obstet 133: 359-364. 
15. Mikdachi H, Arielle Schreck (2018) Robotic surgery in the obese patient: Tips and tricks for the benign gynecologist. Int J Gynecol Clin Pract 2018: 146.

16. Chiu LH, Chen CH, Tu Pei C, et al. (2015) Comparison of robotic surgery and laparoscopy to perform total hysterectomy with pelvic adhesions or large uterus. J Minim Access Surg 11: 87-93.

17. Almeida OD (2013) Robotic hysterectomy strategies in the morbidly obese patient. JSLS 17: 418-422.

18. Gallo T, Kashani S, Patel DA, et al. (2012) Robotic-assisted laparoscopic hysterectomy: outcomes in obese and morbidly obese patients. JSLS 16: 421-427.

19. Aloisi A, Tseng JH, Sandadi S, et al. (2019) Is robotic-assisted surgery safe in the elderly population? An Analysis of gynecologic procedures in patients $\geq 65$ Years Old. Ann Surg Oncol 26: 244-251.

20. Lavoue V, Xing Zeng, Susie Lau, et al. (2014) Impact of robotics on the outcome of elderly patients with endometrial cancer. Gynecologic Oncology 133: 556-562.

21. Silasi D-A, Gallo T, Silasi M, et al. (2013) Robotic versus abdominal hysterectomy for very large uteri. JSLS 17: 400-406.

22. Walid MS, Heaton RL (2009) Laparoscopic extirpation of a 3-kg uterus. Arch Gynecol Obstet 279: 607-608.

23. Leijte E, Blaauw I de, van Workum F, et al. (2019) Robot assisted versus laparoscopic suturing learning curve in a simulated setting. Surg Endosc 34: 3679-3689.

24. Rusch P, Ind T, Kimmig R, et al. (2019) Recommendations for a standardised educational program in robot assisted gynaecological surgery: Consensus from the Society of European Robotic Gynaecological Surgery (SERGS). Facts Views Vis Obgyn 11: 29-41.

25. Advincula AP, Reynolds RK (2005) The use of robot-assisted laparoscopic hysterectomy in the patient with a scarred or obliterated anterior cul-de-sac. JSLS 9: 287-291. 